\title{
The Clinical Benefit of Adjuvant Therapy in Long-Term Survival of Early-Stage Ampullary Carcinoma: A Single Institutional Experience
}

\author{
Ashish Manne ${ }^{\mathrm{a}, \mathrm{d}}$, Haris Hatic ${ }^{\mathrm{b}}$, Peng $\mathrm{Li}^{\mathrm{b}}$, Rojymon $\mathrm{Jacob}^{\mathrm{c}}$, \\ Grant Williams ${ }^{\mathrm{b}}$, Ravi Palurib
}

\begin{abstract}
Background: The role of adjuvant chemotherapy (CT) or combination chemoradiation (CRT) remains uncertain for ampullary carcinoma (AC). In this analysis, we reviewed our institution's experience with early-stage AC.

Methods: AC patients who had definitive surgical intervention at the University of Alabama, Birmingham, between 2005 and 2015, were identified. Clinicopathologic factors and disease statuses were obtained from chart review. The univariate Cox proportional hazard model was conducted for evaluating the parameters associated with overall survival (OS). Kaplan-Meier method and log-rank method were used to compare the time-to-events. We estimated the survival for the patients who had definitive surgery (pancreaticoduodenectomy (PD) or ampullectomy), and followed them up with assessing the influence of adjuvant treatment (chemoradiotherapy or $\mathrm{CT}$ ) alone on the survival in the early-stage (stage I/II) AC.
\end{abstract}

Results: A total of 63 patients had definitive surgery. The median OS and progression-free survival (PFS) for all the patients who had definitive surgery were 40.5 months and 28 months, respectively. Adjuvant treatment was administered in $60 \%$ of patients with early-stage (stage I/II) AC (CT 36\% and CRT 24\%), while 22\% were on surveillance post surgery. The pathological stage $\geq 2$, Lymph node (LN) metastasis, peri-nodal extension (PNE) and peri-pancreatic extension (PPE) were found to be the determinants for poor OS and PFS by univariate analysis. Multiple Cox regression of these variables showed a signifi-

Manuscript submitted July 8, 2020, accepted July 15, 2020

Published online August 15, 2020

aDivision of Medical Oncology, Department of Internal Medicine, The Ohio State University, Columbus, OH, USA

${ }^{b}$ Department of Hematology \& Oncology, University of Alabama, Birmingham, AL, USA

'Department of Radiation Oncology, University of Alabama, Birmingham, AL, USA

${ }^{\mathrm{d} C}$ Corresponding Author: Ashish Manne, Division of Medical Oncology, Department of Internal Medicine, The Ohio State University, B407 Starling Loving Hall, 320 West 10th Avenue, Columbus, OH 43210, USA.

Email: ashish.manne@osumc.edu

doi: https://doi.org/10.14740/jocmr4267 cant influence of PPE and pathological staging on the OS and PFS, respectively. In the early-stage $\mathrm{AC}$ with no high-risk features, adjuvant therapy did not improve the survival over surgery alone (40.5 vs. 51.7 months, $\mathrm{P}=0.93$ ). The addition of radiation to $\mathrm{CT}$ did not yield improved outcome in early-stage cancers. For CRT and CT, OS was 22.8 versus 65.7 months $(\mathrm{P}=0.3975)$, and $\mathrm{PFS}$ was 25.3 versus 65.7 months $(\mathrm{P}=0.4699)$.

Conclusions: In the early-stage AC, adjuvant therapy may not improve the outcome in the short term but may benefit over a long period. It should be considered, especially in patients with adverse risk factors. Radiation therapy may not be useful in managing AC in the adjuvant setting.

Keywords: Ampullary cancer; Cancer of ampulla of Vater; Adjuvant treatment; Early-stage ampullary cancer; Peri-ampullary cancer; Post-operative management of ampullary cancer; Adverse factors for ampullary cancer

\section{Introduction}

Ampullary carcinomas (ACs) arise distal to the bifurcation of the distal common bile duct and pancreatic duct or from the papilla [1]. It is a rare disease in the general population (4 - 6 per million) and is the second most frequent $(6 \%)$ peri-ampullary cancers after pancreatic cancer [2,3]. With the advancements in available imaging technology, their diagnosis (and hence the incidence) is increasing in the last three decades [4]. Close to $20 \%$ of common bile duct obstructions are tumor-related, warranting careful workup for all obstructive jaundice cases [5]. Risk stratification of AC is a challenging task. Conventionally, they are staged by the tumor, node, metastasis (TNM) system of the combined American Joint Committee on Cancer (AJCC)/Union for International Cancer Control (UICC) [6]. Early-stage cancers like stage I/II are expected to have a good prognosis.

Currently there are no guidelines for management of ACs and they are treated in line with pancreatic cancer based on multidisciplinary consensus. Adjuvant therapy is suggested for "perceived" high-risk features including lymph node (LN) metastasis, T3/T4 [7, 8]. Very low incidence of AC and absence 
of randomized clinical trials posed challenges in identifying clinicopathological risk factors associated with poor outcomes in AC. In the present retrospective study, we attempted to identify clinicopathological adverse risk factors in clinical stage I/II AC patients who had definitive surgery (pancreaticoduodenectomy (PD) or ampullectomy) in our hospital.

\section{Materials and Methods}

A retrospective cohort study was done at the University of Alabama, Birmingham, at Birmingham Hospital after receiving appropriate approvals by the Office of the Institutional Review Board to determine the clinical outcomes of early-stage AC. Utilizing billing search engines and Current Procedural Terminology (CPT) codes, ampullary cancer patients managed between the years of January 2005 and December 2015 were identified in the electronic medical records (EMRs). From chart review, demographic data like age, gender, and race, social history like smoking/alcohol use, lab data like albumin and CA19, clinical staging, type of surgery, pathological stage (P stage), adjuvant therapy and performance status were collected. This study was conducted in compliance with all the applicable institutional ethical guidelines for the care, welfare and use of animals.

The clinical staging (cTNM) was based on imaging (computed tomography (CT)/magnetic resonance imaging (MRI) and endoscopic ultrasonography (EUS)) before the surgery. Pathological staging (pTNM) was based on the operative pathology report. It included histological grade of the tumor (from undifferentiated to well-differentiated), margin status, lymphovascular invasion (LVI), peri-neural invasion (PNI), portal vein invasion (PVI), peri-pancreatic soft tissue extension (PPE), peri-nodal extension (PNE), lymph node (LN) metastasis and invasion of adjacent organs. In this study, the AJCC seventh edition (2010) for staging the AC was adopted, which differs significantly from the AJCC eighth edition (2018). In the seventh edition, the node-positive disease was still considered as early-stage (stage IIB), while in the eighth edition, it is considered an advanced stage (stage IIIA). The demographical and oncological characteristics were summarized with means and standard deviations for continuous variables and proportions for categorical variables.

Overall survival (OS) was defined as the time between surgery and death and was censored at the last follow-up date if the patients were still alive. Progression-free survival (PFS) was defined as the time to recurrence post surgery. KaplanMeier method was used to compare the OS or PFS among subgroups, and the $\mathrm{P}$ values were calculated by the log-rank method. The univariate Cox proportional hazard model was conducted for evaluating the predictors of OS and PFS.

A total of 80 patients were retrospectively evaluated. Seventy-four were considered clinically resectable at presentation. However, nine patients did not get definitive surgeries (seven patients were upstaged intra-operatively, and the operations were aborted), and two patients were lost in the follow-up after the surgery. Finally, 63 patients were included in the study cohort. The study cohort included only the patients with a de- finitive surgical intervention who received adjuvant therapy or surveillance alone. Adjuvant therapy was given in the form of concurrent chemoradiation (CRT) or chemotherapy (CT) alone. For the analysis of adjuvant therapy, we included only the $\mathrm{AC}$ that remained as early-stage (P stage I/II) and excluded the $\mathrm{AC}$ that got upstaged post surgery (P stage III/IV).

\section{Results}

The median age of the cohort was 61 years, with 51\% (32/63) women and predominantly whites $(82.5 \%)$. Only $11 \%$ of the study population had a history of pancreatitis, while $23.8 \%$ had diabetes mellitus. Smoking and alcohol history was present in $42.9 \%$ and $19.1 \%$, respectively. Out of 63 patients in the cohort, $87 \%$ remained early-stage after surgery, while eight patients got upstaged (seven stage III and one stage IV). The rest of the baseline characteristics are summarized in Table 1.

\section{Survival}

The median OS of the entire cohort of 80 patients was 40.5 months (Fig. 1a). The survival rate of the whole cohort was $94.4 \%$ at the end of the first year and dropped to $19 \%$ in the 10th year. The median PFS of the cohort was 28 months (Fig. $1 \mathrm{~b})$, and PFS rate was close to $13 \%$ at the end of the 10 years.

The univariate analysis showed a statistically significant influence of pathological staging (P stage) $\geq 2$, LN metastasis, PNE, and PPE on the OS and PFS (Table 2). For OS, multiple Cox regression, including LN metastasis, PPE, PNE, and $P$ stage, only suggested the significant effect of PPE, partially due to the small sample size and multicollinearity. For PFS, multiple Cox regression, including LN metastasis, PPE, PNE, and P stage, only suggested the significant effect of P stage, partially due to the small sample size and multicollinearity.

In the early-stage AC group, the median OS and PFS were 44.1 months and 40.5 months, respectively. In stage I AC, median OS and PFS were close to 7 years.

\section{Influence of adjuvant therapy in early-stage ampullary cancer}

Thirty-three patients received either adjuvant treatment, while 12 patients were put on surveillance. Adjuvant therapy was offered to patients with $\mathrm{LN}$ disease and/or positive margins.

\section{Surgery alone versus surgery plus adjuvant treatment}

There was no significant difference in the median OS for the patients who were on surveillance compared to those who received adjuvant therapy (40.5 vs. 51.7 months, $\mathrm{P}=0.93)$. Fiveyear survival rate was worse in the adjuvant therapy group ( $43 \%$ vs. $50 \%$ ), but the 10 -year survival rate was $30 \%$ in them, and none of the patients in the surveillance group were alive (at 10-year mark) (Fig. 2a). 
Table 1. Demographical and Oncological Characteristics of Patients

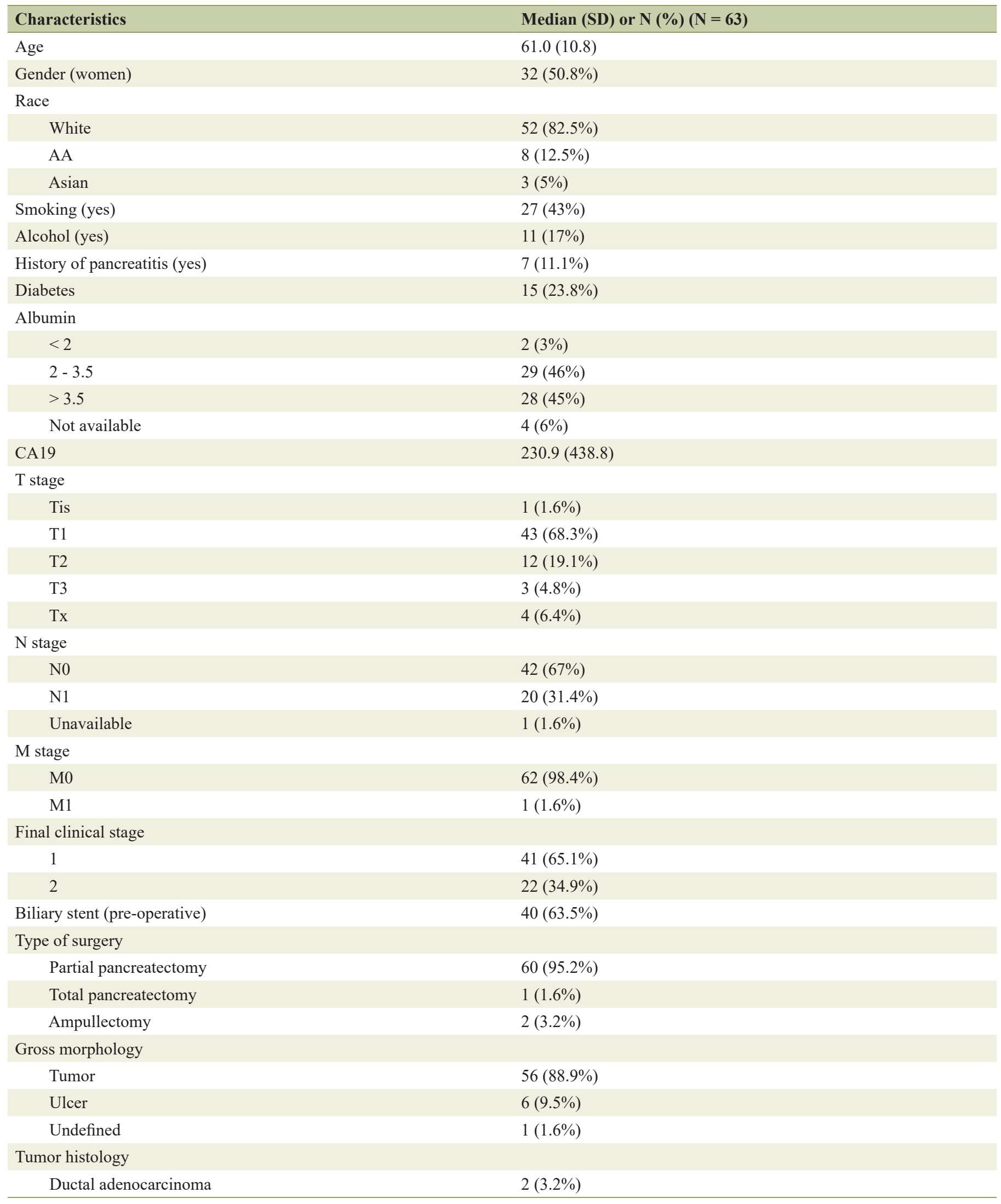


Table 1. Demographical and Oncological Characteristics of Patients - (continued)

\begin{tabular}{|ll|}
\hline Characteristics & Median (SD) or N (\%) $\mathbf{~} \mathbf{~ = ~ 6 3 ) ~}$ \\
\hline \multicolumn{1}{|c|}{ Signet-ring cell carcinoma } & $2(3.2 \%)$ \\
$\quad$ Adenocarcinoma (not otherwise specified) & $57(93.6 \%)$ \\
Margin status & \\
$\quad$ Involved & $3(4.8 \%)$ \\
$\quad$ Uninvolved & $58(92 \%)$ \\
$\quad$ N/A & $2(3.2 \%)$ \\
Lymphovascular invasion (yes) & $15(23.8 \%)$ \\
Peri-neural invasion (yes) & $10(15.9 \%)$ \\
Peri-pancreatic soft tissue extension (yes) & $12(19.1 \%)$ \\
Peri-nodal extension (yes) & $33(52.4 \%)$ \\
Treatment modality & \\
Adjuvant chemotherapy & $25(39 \%)$ \\
Adjuvant chemotherapy + radiation & $13(22 \%)$ \\
No adjuvant therapy & $25(39 \%)$ \\
Post-operative therapy for advanced stage & $5(7 \%)$ \\
Unknown management & $13(21 \%)$ \\
\hline
\end{tabular}

SD: standard deviation; AA: Asian Americans.

The median PFS followed the same trend as the OS. Patients who received adjuvant therapy did not have any favorable outcome over the surgery alone (30.6 vs. 51.7 month, $\mathrm{P}=$ 0.71 ) in the first 5 years. However, at the 10 -year milestone, $20 \%$ of the patients in the adjuvant therapy group had recurrence while all the patients in the surveillance group either died (Fig. 2b).

\section{CT versus CRT}

To assess the benefit of adding radiation to CT, the CRT group was compared with the CT group. Radiation was offered to patients with positive margins and LN disease at the physician's discretion. In the CT group, the majority of them got capecitabine alone. Other CT agents like gemcitabine alone, 5-fluorouracil (5-FU) alone, gemcitabine/capecitabine, and

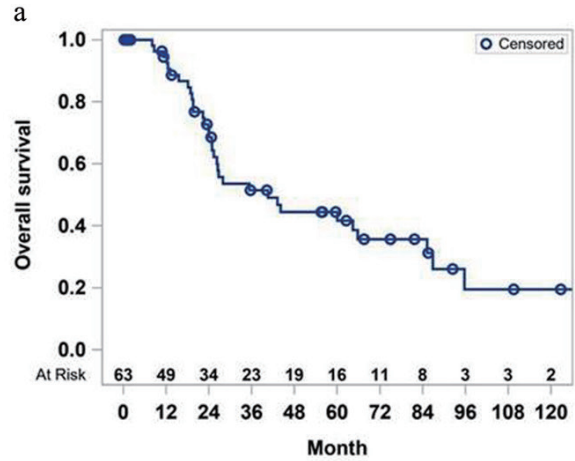

gemcitabine/cisplatin were used in some patients. In the CRT group, capecitabine and 5-FU were used as radiosensitizers.

For the early-stage AC, adjuvant radiation did not show survival benefit (22.8 vs. 65.7 months, $\mathrm{P}=0.39$ ) (Fig. 3a). Even though the survival rate was higher in CT group at 5-year mark, over 10 years, that difference narrowed to $5 \%$. Similarly, PFS was better in the CT group (25.3 vs. 65.7 month, $\mathrm{P}=0.46$ ) (Fig. 3b).

\section{Discussion}

The definitive treatment for early-stage AC is surgery [1]. Overall the clinical outcomes rely on post-operative factors including pathological staging and identification of the high features such as size of the tumor, margin status, pathological staging, PVI, PNI, PNE, and PPE [9]. AC associated with

b

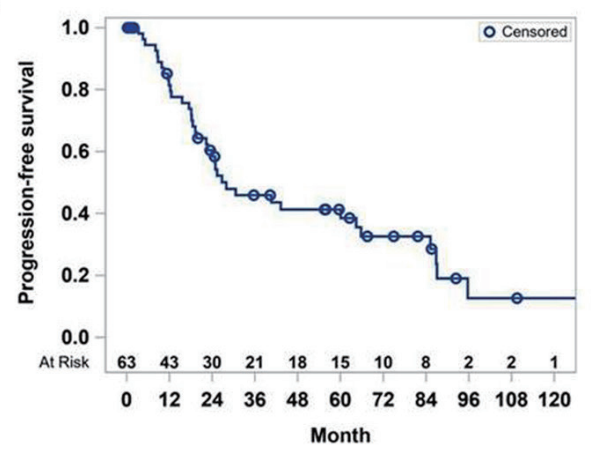

Figure 1. Overall survival (a) and progression-free survival (b) of study cohort. 
Table 2. Univariate Analyses of Overall Survival and Progression-Free Survival

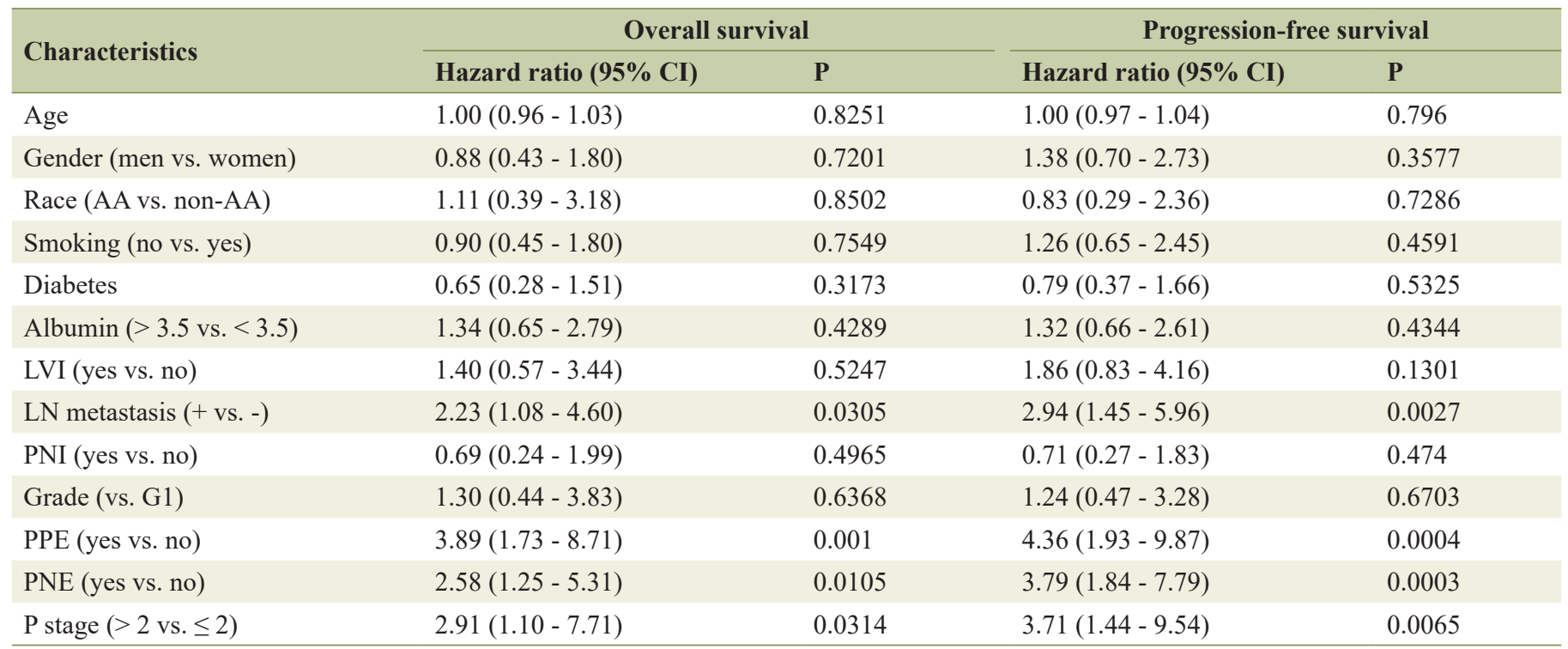

Cl: confidence interval; PPE: peri-pancreatic extension; LN: lymph node; PNE: peri-nodal extension; PNI: peri-neural invasion; P stage: pathological stage; LVI: lymphovascular invasion; AA: Asian Americans.

hereditary polyposis syndromes like familial adenomatous polyposis (FAP) and hereditary nonpolyposis colorectal cancer (HNPCC) present at an earlier age than sporadic cases $[10,11]$. Molecular studies show improved survival in $\mathrm{AC}$ with microsatellite instability (MSI), while immunohistological studies did not show any correlation with survival [12]. Risk stratification based on molecular profiles with mutations like TP53, K-RAS, APC, ELF-3, WNT, PI3K, and
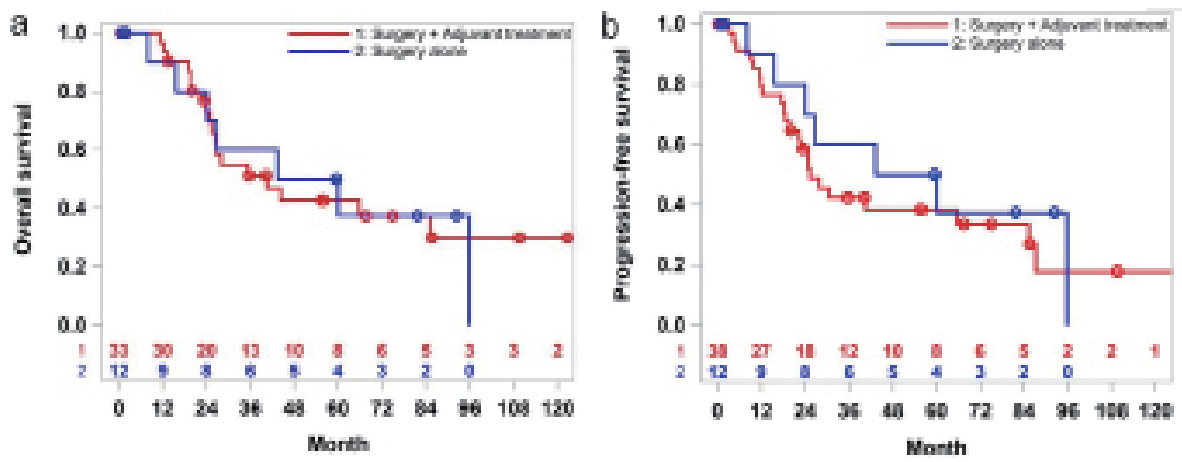

Figure 2. Overall survival (a) and progression-free survival (b) of stages 1 and 2 patients: surgery alone versus surgery plus adjuvant treatment.
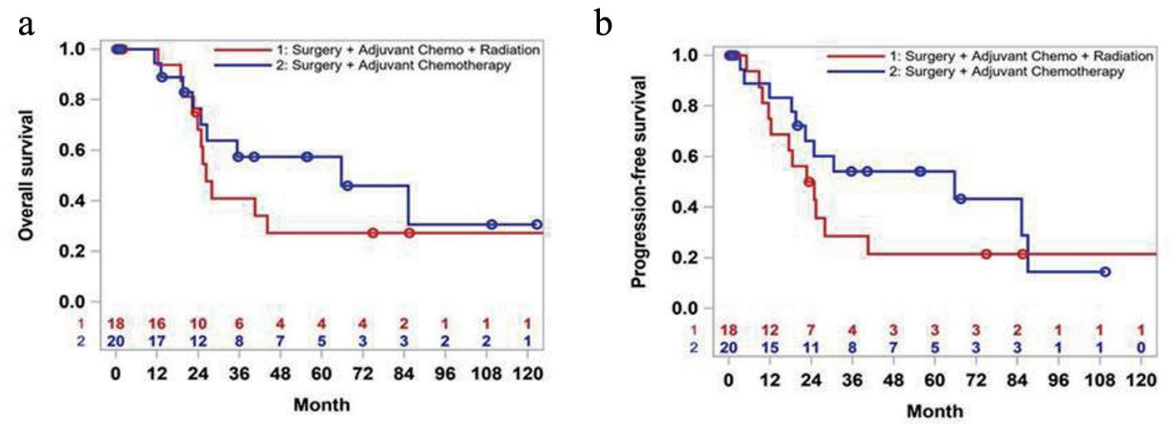

Figure 3. Overall survival (a) and progression-free survival (b) of patients in stages 1 and 2: surgery plus chemotherapy versus surgery plus chemotherapy plus radiation. 
ERBB2 has been proposed, but none of them are popular in current clinical practice $[9,13-16]$.

In this study, the focus was on recognizing the adverse factors in early-stage (clinical) AC who get upfront definitive surgery, the role of adjuvant treatment in the early-stage (pathological) AC, and also the benefit of radiation among the patients who proceed to get adjuvant therapy. These results will help treating physicians in making informed decisions.

\section{Risk stratification of $\mathrm{AC}$}

One of the early studies in AC out of Memorial Sloan-Kettering Cancer Center showed that clear margins and absence of LN metastasis conferred better prognosis while the other pathological factors like tumor size, T staging, histology, PNE, and PVI had no impact on the outcome of the patients who get definitive surgery (Whipple's) in AC [1]. In another study with more than 5,000 cases, pathological features like histology type, grade, P stage, and pre-existing ampullary adenomas were considered for risk stratification. Low-grade papillary carcinomas had a better prognosis than a mucinous or not-otherwise specified (NOS) adenocarcinoma. Cancers developing from pre-existing adenomas had better survival than de novo cancers [4]. Association of poor outcomes with PNI and LVI in AC patients was also established in another retrospective study by Chavez et al [17].

In this study, PPE and P stage ( $\leq 2$ or $>2$ ) had a significant impact on the OS and PFS. Other adverse risk factors like LN metastasis and PNE seem to have some effect on the survival. Age of diagnosis, gender, race, smoking history, and diabetes have no bearing on the survival. These are in line with previously reported studies.

\section{Role of adjuvant therapy in early-stage AC}

In the early-stage $\mathrm{AC}$, there are no clear guidelines on adjuvant therapy. The consensus was to offer it in AC with "high-risk" features like nodal metastasis and invasion of the pancreas by the tumor (T3/T4) $[7,18]$. In a study out of Duke University, the authors argued that adjuvant therapy should be given even to stage I AC [8].

In the present study, when compared to the adjuvant therapy (CT or CRT) group, the surveillance group did better in the first 5 years. This survival advantage did not consolidate over the 10 years, and in fact, it was worse: the survival rate was $30 \%$ in the adjuvant therapy group while none of the surveillance group patients were alive. Pending validation by larger prospective trials, there seems to be role of adjuvant therapy in selected sub-population with early-stage cancer, likely those with presumed high-risk features ( $\mathrm{pT} 2, \mathrm{LN}$, and positive margins). Even though few retrospective studies suggest the impact of histologic classification (pancreaticobiliary vs. intestinal) on outcomes, there are no marked differences in their management currently [19].

In the current clinical practice, there is still hesitancy in administering adjuvant therapy (CT or CRT) in early-stage AC. Extrapolating the data from the pancreatic cancer trials, the benefit of adjuvant CT is advocated [20]. The fluorouracil, leucovorin, oxaliplatin and irinotecan (FOLFIRINOX) combination is widely used in suitable (good performance status) patients if adjuvant CT alone is considered [21]. Gemcitabine alone or its combination with capecitabine is reasonable in patients with poor performance status $[20,22]$.

The benefit of adjuvant radiation alone or in combination with CT is under scrutiny. Some studies were able to show the benefit of just radiation therapy (without a combination of CT) in early-stage cancers [23]. The European societies even advise against radiation (based on European Study Group for Pancreatic Cancer (ESPAC)-1 trial), but in the USA, it is still used in selected cases [24]. If CRT is considered, 5-FU or capecitabine is administered during radiation. Usually, it follows 4 months of systemic therapy. In this study, the addition of radiation to $\mathrm{CT}$ did not improve the outcome significantly, and it should be avoided and the patients should be protected from any extra morbidity.

Small sample size and retrospective model are the major limitations of this study, but the results are in accordance with other retrospective studies in the literature. We have close to 10 years of follow-up, which is one of the unique parts of this study.

\section{Conclusions}

Risk stratification of $\mathrm{AC}$ is essential in formulating the best management plan for early-stage cancers. Adjuvant therapy should be considered in patients with high-risk features including post-operative upstaging, PPE, LN metastasis, and PNE. The benefit of adding CRT to CT is inconclusive and needs to be validated in randomized trials. It can be considered in selected patients with high-risk features. Given the dismal outcomes of even early-stage AC who have successful curative surgeries, there is an unmet need for multi-center, randomized trials to guide adjuvant therapy.

\section{Acknowledgments}

None to declare.

\section{Financial Disclosure}

None to declare.

\section{Conflict of Interest}

None to declare.

\section{Informed Consent}

Not applicable. 


\section{Author Contributions}

The study was designed by authors AM and RP. Data were collected and manuscript was drafted by AM. RP supervised the manuscript preparation and did critical editing. $\mathrm{HH}$ participated in data collection; PL contributed to statistical analysis; $\mathrm{RJ}$ and GW did critical editing of the manuscript.

\section{Data Availability}

The authors declare that data supporting the findings of this study are available within the article.

\section{References}

1. Howe JR, Klimstra DS, Moccia RD, Conlon KC, Brennan MF. Factors predictive of survival in ampullary carcinoma. Ann Surg. 1998;228(1):87-94.

2. Ruemmele P, Dietmaier W, Terracciano L, Tornillo L, Bataille F, Kaiser A, Wuensch PH, et al. Histopathologic features and microsatellite instability of cancers of the papilla of vater and their precursor lesions. Am J Surg Pathol. 2009;33(5):691-704.

3. Benhamiche AM, Jouve JL, Manfredi S, Prost P, Isambert N, Faivre J. Cancer of the ampulla of Vater: results of a 20-year population-based study. Eur J Gastroenterol Hepatol. 2000;12(1):75-79.

4. Albores-Saavedra J, Schwartz AM, Batich K, Henson DE. Cancers of the ampulla of vater: demographics, morphology, and survival based on 5,625 cases from the SEER program. J Surg Oncol. 2009;100(7):598-605.

5. Palazzo L. Staging of ampullary carcinoma by endoscopic ultrasonography. Endoscopy. 1998;30(Suppl 1):A128131.

6. Amin MB, Greene FL, Edge SB, Compton CC, Gershenwald JE, Brookland RK, Meyer L, et al. The Eighth Edition AJCC Cancer Staging Manual: Continuing to build a bridge from a population-based to a more "personalized" approach to cancer staging. CA Cancer J Clin. 2017;67(2):93-99.

7. Lee JH, Whittington R, Williams NN, Berry MF, Vaughn DJ, Haller DG, Rosato EF. Outcome of pancreaticoduodenectomy and impact of adjuvant therapy for ampullary carcinomas. Int J Radiat Oncol Biol Phys. 2000;47(4):945-953.

8. Zhong J, Palta M, Willett CG, McCall SJ, McSherry F, Tyler DS, Uronis HE, et al. Patterns of failure for stage I ampulla of Vater adenocarcinoma: a single institutional experience. J Gastrointest Oncol. 2014;5(6):421-427.

9. Mandelker D, Zhang L, Kemel Y, Stadler ZK, Joseph V, Zehir A, Pradhan N, et al. Mutation detection in patients with advanced cancer by universal sequencing of cancerrelated genes in tumor and normal DNA vs guidelinebased germline testing. JAMA. 2017;318(9):825-835.

10. Jagelman DG, DeCosse JJ, Bussey HJ. Upper gastrointestinal cancer in familial adenomatous polyposis. Lan- cet. 1988;1(8595):1149-1151.

11. Nakase A, Matsumoto Y, Uchida K, Honjo I. Surgical treatment of cancer of the pancreas and the periampullary region: cumulative results in 57 institutions in Japan. Ann Surg. 1977;185(1):52-57.

12. Sessa F, Furlan D, Zampatti C, Carnevali I, Franzi F, Capella C. Prognostic factors for ampullary adenocarcinomas: tumor stage, tumor histology, tumor location, immunohistochemistry and microsatellite instability. Virchows Arch. 2007;451(3):649-657.

13. Pea A, Riva G, Bernasconi R, Sereni E, Lawlor RT, Scarpa A, Luchini C. Ampulla of Vater carcinoma: Molecular landscape and clinical implications. World J Gastrointest Oncol. 2018;10(11):370-380.

14. Yachida S, Wood LD, Suzuki M, Takai E, Totoki Y, Kato M, Luchini C, et al. Genomic sequencing identifies ELF3 as a driver of ampullary carcinoma. Cancer Cell. 2016;29(2):229-240.

15. Gingras MC, Covington KR, Chang DK, Donehower LA, Gill AJ, Ittmann MM, Creighton CJ, et al. Ampullary cancers harbor ELF3 tumor suppressor gene mutations and exhibit frequent WNT dysregulation. Cell Rep. 2016;14(4):907-919.

16. Pinto P, Peixoto A, Santos C, Rocha P, Pinto C, Pinheiro M, Leca L, et al. Analysis of founder mutations in rare tumors associated with hereditary breast/ovarian cancer reveals a novel association of BRCA2 mutations with ampulla of vater carcinomas. PLoS One. 2016;11(8): 0161438

17. Chavez MT, Sharpe JP, O'Brien T, Patton KT, Portnoy DC, VanderWalde NA, Deneve JL, et al. Management and outcomes following pancreaticoduodenectomy for ampullary adenocarcinoma. Am J Surg. 2017;214(5):856861.

18. Krishnan S, Rana V, Evans DB, Varadhachary G, Das P, Bhatia S, Delclos ME, et al. Role of adjuvant chemoradiation therapy in adenocarcinomas of the ampulla of vater. Int J Radiat Oncol Biol Phys. 2008;70(3):735743.

19. Chandrasegaram MD, Gill AJ, Samra J, Price T, Chen J, Fawcett J, Merrett ND. Ampullary cancer of intestinal origin and duodenal cancer - A logical clinical and therapeutic subgroup in periampullary cancer. World J Gastrointest Oncol. 2017;9(10):407-415.

20. Oettle H, Post S, Neuhaus P, Gellert K, Langrehr J, Ridwelski K, Schramm H, et al. Adjuvant chemotherapy with gemcitabine vs observation in patients undergoing curative-intent resection of pancreatic cancer: a randomized controlled trial. JAMA. 2007;297(3):267-277.

21. T C, et al. FOLFIRINOX or gemcitabine as adjuvant therapy for pancreatic cancer. The New England Journal of Medicine. 2018;379(25).

22. Neoptolemos JP, Palmer DH, Ghaneh P, Psarelli EE, Valle JW, Halloran CM, Faluyi O, et al. Comparison of adjuvant gemcitabine and capecitabine with gemcitabine monotherapy in patients with resected pancreatic cancer (ESPAC-4): a multicentre, open-label, randomised, phase 3 trial. Lancet. 2017;389(10073):1011-1024.

23. Narang AK, Miller RC, Hsu CC, Bhatia S, Pawlik TM, 
Laheru D, Hruban RH, et al. Evaluation of adjuvant chemoradiation therapy for ampullary adenocarcinoma: the Johns Hopkins Hospital-Mayo Clinic collaborative study. Radiat Oncol. 2011;6:126.
24. Neoptolemos JP, Stocken DD, Friess H, Bassi C, Dunn JA, Hickey H, Beger H, et al. A randomized trial of chemoradiotherapy and chemotherapy after resection of pancreatic cancer. N Engl J Med. 2004;350(12):1200-1210. 